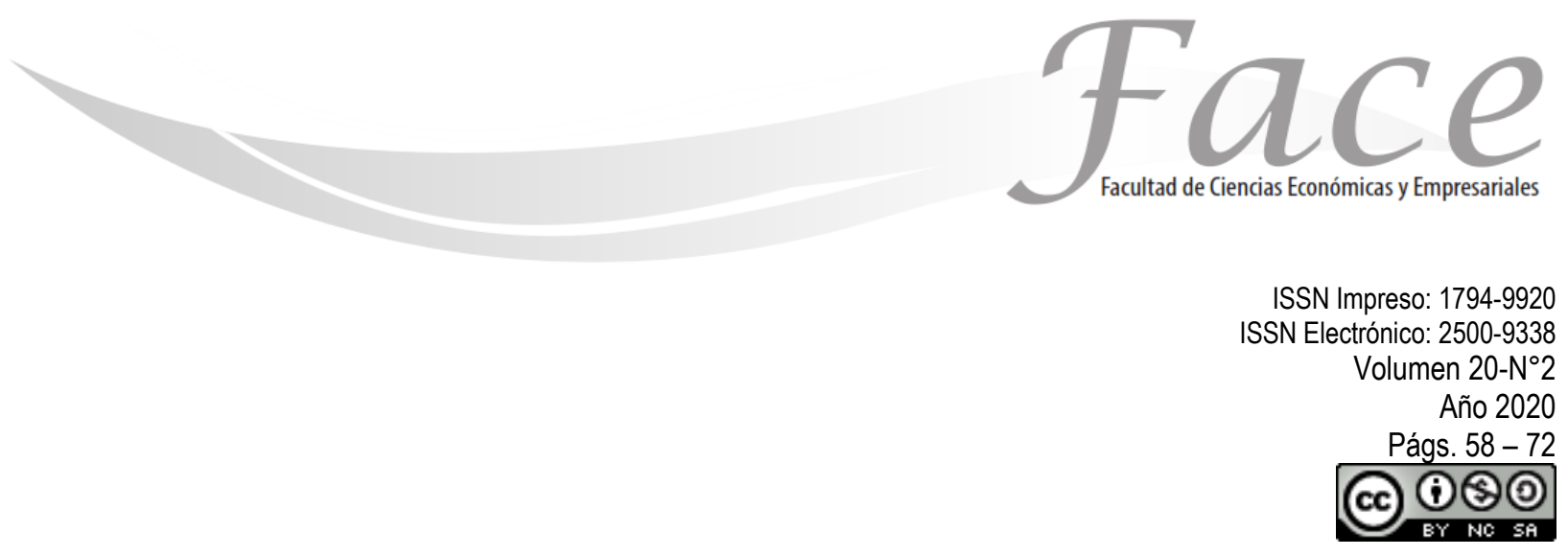

\title{
REVISIONES METODOLÓGICAS PARA EL ESTUDIO DE LA SUSTENTABILIDAD EN EL TURISMO
}

\author{
Ana Karen Flores-Pozos* \\ Enlace ORCID: https://orcid.org/0000-0002-8032-7809 \\ Carlos Alberto Pérez-Ramírez* \\ Enlace ORCID: https://orcid.org/0000-0002-8074-2391 \\ Ruth Moreno-Barajas*** \\ Enlace ORCID: hthps:/orid.org/0000-0002-2078-037X
}

Fecha de Recepción: Septiembre 7 de 2020

Fecha de Aprobación: Diciembre 17 de 2020

\section{Resumen:}

El turismo ha tenido un crecimiento exponencial en los últimos años, gracias a sus múltiples beneficios, no obstante, existen diversas problemáticas ambientales, sociales y económicas que surgen por la mala gestión de la actividad. Por esa razón, es importante impulsar el desarrollo desde un enfoque sustentable con la finalidad de lograr un equilibrio entre los distintos ámbitos. En ese sentido, es necesario identificar las metodologías que se han empleado para la evaluación de la sustentabilidad, y retomarlas como una línea de investigación en los estudios ambientales del turismo. Por ello, el objetivo de la investigación fue realizar una revisión de la literatura en materia de evaluación de la sustentabilidad en el turismo, publicados en diversas revistas indizadas, disponibles en hemerotecas científicas y bases de datos como Scopus, Elsevier, Redalyc, Scielo, entre otros, durante el periodo 2004-2020. Se identificaron 52 artículos relacionados al tema de interés, destacando que aún no se han realizado estudios que permitan comprender los alcances del desarrollo de la actividad turística desde la posición de los actores locales como eje central en el proceso de desarrollo del turismo, y de esta forma, poder dar soluciones que reviertan el impacto actual en el ambiente, la economía y el ámbito social

Palabras clave: Turismo, Sustentabilidad, Actores Locales

*Maestría en Ciencias Ambientales. Estudiante del Doctorado de Ciencias Ambientales. Facultad de Química, México. Universidad Autónoma del Estado de México, México. Contacto: akarenfp92@gmail.com

** Doctor en Ciencias Ambientales. Profesor Investigador de la Facultad de Planeación Urbana y Regional, México. Universidad Autónoma del Estado de México, México. Contacto: caperezr@uaemex.mx

*** Doctora en Ciencias Agropecuarias y Recursos Naturales. Profesora Investigadora de la Facultad de Planeación Urbana y Regional, México. Universidad de Autónoma del Estado de México, México. Contacto: rmorenob@uaemex.mx 


\title{
METHODOLOGICAL REVIEWS FOR THE STUDY OF SUSTAINABILITY IN TOURISM
}

\begin{abstract}
:
Tourism has grown exponentially in recent years, thanks to its many benefits, however, there are various environmental, social, and economic problems that arise from poor management of the activity. For this reason, it is important to promote development from a sustainable approach to achieve a balance between the different areas. In this sense, it is necessary to identify the methodologies that have been used for the evaluation of sustainability, and retake them as a line of research in environmental studies of tourism. Therefore, the objective of the research was to carry out a review of the literature on the evaluation of sustainability in tourism, published in various indexed journals, available in scientific newspaper libraries and databases such as Scopus, Elsevier, Redalyc, Scielo, among others, during the period 20042020. 52 articles related to the topic of interest were identified, highlighting that studies have not yet been carried out that verify the scope of the development of tourism activity from the position of local actors as the central axis in the tourism development process, and thus, to be able to provide solutions that reverse the current impact on the environment, the economy and the social sphere.
\end{abstract}

Keywords: tourism, sustainability, local actors.

\section{REVISÕES METODOLÓGICAS PARA O ESTUDO DA SUSTENTABILIDADE NO TURISMO}

\begin{abstract}
Abstrato:
O turismo tem crescido exponencialmente nos últimos anos, graças aos seus inúmeros benefícios, no entanto, vários são os problemas ambientais, sociais e econômicos decorrentes da má gestão da atividade. Por isso, é importante promover o desenvolvimento a partir de uma abordagem sustentável para alcançar o equilíbrio entre as diferentes áreas. Nesse sentido, é necessário identificar as metodologias que vêm sendo utilizadas para a avaliação da sustentabilidade, e retomá-las como linha de pesquisa em estudos ambientais do turismo. Portanto, o objetivo da pesquisa foi realizar uma revisão da literatura sobre avaliação da sustentabilidade no turismo, publicada em diversos periódicos indexados, disponíveis em bibliotecas de jornais científicos e bases de dados como Scopus, Elsevier, Redalyc, Scielo, entre outros. durante o período 2004-2020. Foram identificados 52 artigos relacionados ao tema de interesse, destacando-se que ainda não foram realizados estudos que verifiquem o alcance do desenvolvimento da atividade turística a partir da posição dos atores locais como eixo central do processo de desenvolvimento turístico e, portanto, para ser capaz de fornecer soluções que revertam o impacto atual no meio ambiente, na economia e na esfera social.
\end{abstract}

Palavras-chave: turismo, sustentabilidade, atores locais. 


\section{INTRODUCCIÓN:}

La actividad turística es un componente fundamental en la economía internacional, que en los últimos años ha presentado un crecimiento exponencial en países en vías de desarrollo, trayendo diversos beneficios como la generación de empleo, obras de infraestructura, desarrollo de servicios públicos como transporte, salud, educación; entre otros; impactando en la calidad de vida de los habitantes.

Sin embargo, a pesar de los múltiples beneficios sociales y económicos, el desarrollo de esta actividad trae como consecuencias problemáticas ambientales como la contaminación de cuerpos de agua (Reul, Febles-patrón, \& Nava-galindo, 2015), proliferación de residuos sólidos (Romero, 2010), extracción de flora y fauna, deforestación (César, Anaya, \& Ruiz, 2017); así como problemáticas económicas y sociales (Olivier, Rodríguez, López, Cañedo, \& Valera, 2005) que comprometen el funcionamiento de la actividad.

Por lo mencionado anteriormente, es necesario impulsar el desarrollo de la actividad desde el enfoque de la sustentabilidad, con la finalidad de lograr un equilibrio entre el aprovechamiento de los recursos, el crecimiento económico y el bienestar social, asimismo se ha presentado la necesidad de realizar diversas contribuciones y estudios que permitan delinear una metodología para la evaluación de la sustentabilidad con el propósito de conocer las condiciones actuales y futuras de la actividad con múltiples enfoques y perspectivas.

En ese sentido, el objetivo de la presente investigación es realizar una revisión de la literatura en materia de evaluación de la sustentabilidad, para ello se ha indagado en estudios de diversas revistas indizadas, durante el periodo 2004-2020. Este documento se divide en tres apartados. En primera parte se expone un acercamiento teórico a la sustentabilidad, así como una recopilación de los indicadores fomentados por organismos internacionales. La segunda parte se presenta la metodología que se adoptó para llevar a cabo la selección de los artículos analizados.
La tercera etapa presenta un análisis en función de la orientación temática, los enfoques metodológicos y el espacio físico donde se desarrollan dichas investigaciones y estudios previos. Finalmente, se presentan las conclusiones.

\section{MARCO TEÓRICO:}

\section{Sustentabilidad}

Desde los años sesenta, hasta la fecha los temas ambientales han tomado relevancia en la sociedad, a pesar de ello, las actividades antropogénicas y el sistema económico actual, han traído como consecuencia la pérdida de recursos que ha resultado en un sistema ecológicamente insostenible. No obstante, a partir de la Conferencia de Estocolmo se plantea como asunto central que los problemas ambientales se desarrollan de acuerdo con las diferencias territoriales, culturales y económicas. Además, las estrategias de desarrollo convencional empezaron a ser deficientes y se hicieron notables diversas problemáticas como la brecha de desigualdad, reflejada en aumento de la pobreza y el acceso a servicios, por dichas razones la política internacional se vio en la necesidad de estructurar un nuevo modelo de desarrollo.

Por esta razón en 1972 en la Conferencia de las Naciones Unidas sobre Medio Ambiente Humano, celebrada en Estocolmo se planea una visión crítica del modelo predominante, este evento marca un antes y un después al plantear un acercamiento entre la conservación ambiental y los objetivos tradicionales de desarrollo. No obstante, en el año 1987 se pública el informe Brundtland o "Nuestro futuro común" donde se plantea un nuevo modelo de desarrollo donde se tome en cuenta las distintas problemáticas mencionadas anteriormente, para ello toman 
como ejes principales impulsar el crecimiento económico, preservar el ambiente y lograr la equidad social, utilizando por primera vez el término sustentabilidad, definida como "la satisfacción de las necesidades de la generación presente sin comprometerse la capacidad de las generaciones futuras para satisfacer su propias necesidades" (ONU, 2018).

A partir de ese momento se empezaron a incorporar más dimensiones haciendo que el concepto sea más complejo y multidimensional pretendiendo relacionar diversas temáticas relacionadas con aspectos ambientales, económicos y sociales. Asimismo, existen autores como Nieves (1998) que mencionan que la definición no ha logrado ser aceptada; asimismo se ha convertido de un enfoque técnico y físico a uno más social y político, de la misma forma Beaucage (2007) y Zizumbo y Monterroso (2017) considera que el concepto ha surgido desde el sistema capitalista generando estrategias que se encuentran enmarcadas en la tendencia económica, visualizando a la sustentabilidad como un discurso político.

\section{Evaluación de la sustentabilidad en el turismo.}

A lo largo de todo este año se han desarrollado diversos métodos para evaluar la sustentabilidad y de esta manera, poder delinear acciones y políticas acorde a solucionar las problemáticas detectadas, en ese sentido, Speelman y colaboradores (2007) clasifican la evaluación de la sustentabilidad en tres grupos:

a) Generar lista de indicadores. Este grupo se basa en aplicar indicadores de distintos organismos internacionales o nacionales y adecuarlo a distintos contextos.

b) Determinación de índices de sustentabilidad. Se estructura un índice basado en la experiencia del autor en cuento la selección, ponderación y agregación de indicadores, este método permite obtener un valor numérico.

c) Marco de evaluación. Se construye a través de criterios e indicadores más específicos

Correspondiente al primer grupo de evaluación de la sustentabilidad, a nivel mundial existen diversas metodologías propuestas por organismo internaciones como el índice de desarrollo humano (IDH) (PNUD, 2018), los objetivos de desarrollo sustentable (ODS) (UNSD, 2016) y el propuesto por la Comisión Económica de América Latina y el Caribe (CEPAL) (Schuschny \& Soto, 2009) y para el caso especial de la actividad turística destaca los indicadores delineados por la Organización Mundial del Turismo (OMT) (2000) y la Asociación de Estado del Caribe (AEC) (AEC, 2012); que posteriormente los de acuerdo con el autor se van a seleccionar e integrar para poder obtener un valor numérico.

Posteriormente para el grupo tres, como ejemplo se encuentra el Modelo Presión-EstadoRespuesta (PER), donde se categoriza y clasifica cuestiones ambientales interrelacionadas a actividades sociodemográficas. Que de forma general categoriza a las actividades antropogénicas como aquellas que ejercen presión en el sistema $(P)$, trayendo como consecuencia la modificación de las características de los recursos naturales, es decir Estado (E) y que finalmente se evidencian la respuesta de la sociedad a tales transformaciones (OCDE, 2000). Otro ejemplo es la creación de un Marco para la Evaluación de Sistemas de Manejo de Recursos Naturales (MESMIS) incorporando indicadores de sustentabilidad (UNAM, 2020).

\section{Turismo y sustentabilidad}

Como consecuencia de las diferentes cumbres internacionales en torno a las problemáticas ambientales, sociales y económicos; el turismo en la década de los noventa empezó a plantear nuevas vías de desarrollo, para ello en 1993, la Organización Mundial del Turismo define al 
turismo sustentable como aquel que responde a las necesidades de los turistas actuales y las regiones receptivas, protegiendo y agrandando las oportunidades futuras. Se le representa como rector de todos los recursos de modo que las necesidades económicas, sociales y estéticas puedan ser satisfechas manteniendo la integridad cultural de los procesos ecológicos, esenciales, la diversidad biológica y los sistemas en defensa de la vida (OMT, 2019). Estableciendo como principios esenciales (llustración 1):

\section{Ilustración 1 Principios del turismo sustentable}

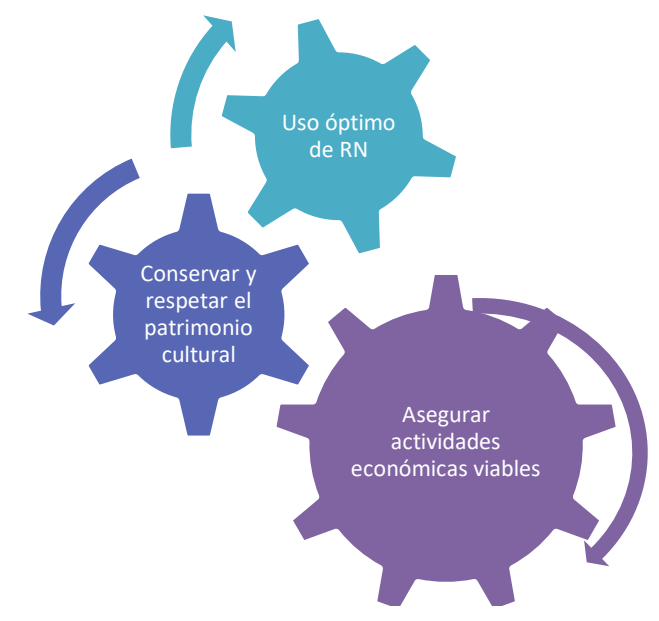

Fuente: Constructo del autor con base en OMT (2019)

\section{METODOLOGÍA:}

El presente estado del arte adoptó como método de investigación un análisis de contenido, que consistió en la revisión de manera sistemática mediante un enfoque cualitativo, donde se identificaron las siguientes categorías orientación temática, enfoque metodológico y espacio físico, en su conjunto se abordó la evaluación de la sustentabilidad desde diferentes perspectivas. Este análisis se conformó de 52 artículos consultados. Los cuales se obtuvieron de repositorios de acceso abierto tales como ScieELO, Elsevier, la Red de Revistas Científicas de América Latina y el Caribe, España y Portugal (Redalyc), entre otros; que concentran un número importante de revistas de diversas disciplinas.

El procedimiento de búsqueda consistió en filtrar los artículos de acuerdo con las palabras clave: evaluación sustentabilidad, indicadores sustentabilidad, turismo y en lengua inglesa se utilizaron los términos sustainability evaluation, sustainability indicators, Tourism (Gráfico 1); para la selección de la información se tomó en cuenta la actualidad de los textos, en los cuales se consideró el período 2000-2020 (Gráfico 1).

\section{Gráfico 1 Frecuencia de publicación por idioma}

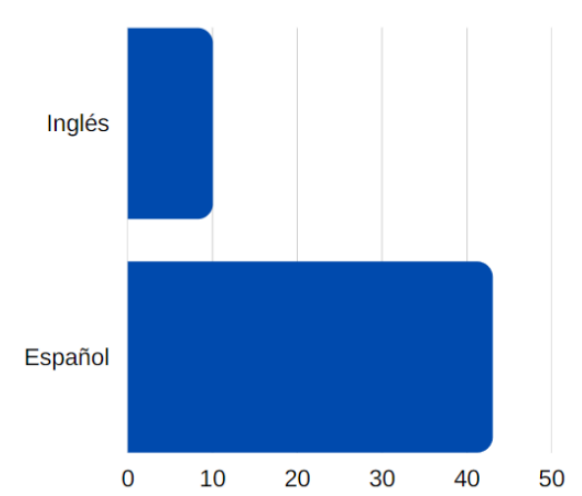

Fuente: Constructo del autor con base en la información recopilada

\section{Gráfico 2 Frecuencia de publicación por año}

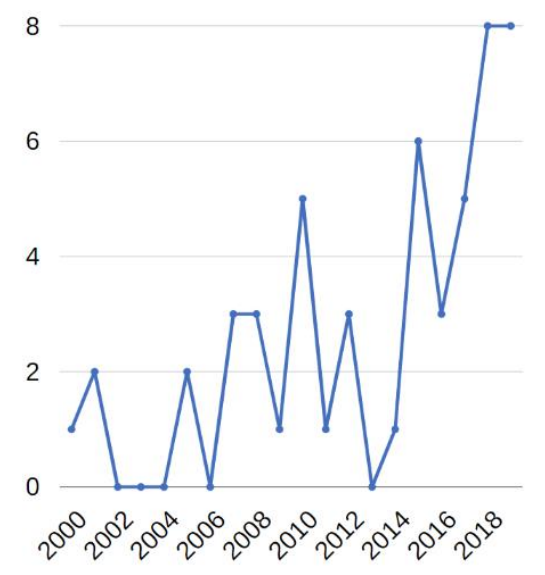

Fuente: Constructo del autor con base en la información recopilada 
Posteriormente se clasificó y analizó la información de acuerdo con las categorías mencionadas anteriormente.

\section{RESULTADOS:}

La evaluación de la sustentabilidad en el turismo es un tema de gran relevancia, pues gracias a ello se muestra un panorama integral que permita identificar las áreas de oportunidad que mejoren las condiciones de desarrollo de las comunidades en las cuales se establece la actividad, por esa razón, se ha convertido en un tema recurrente en la línea de investigación de los estudios ambientales del turismo. En este apartado se plasma la contribución científica de 52 documentos, clasificado en tres distintos enfoques, primero se encuentra el espacio físico que identifica los lugares en los cuales se ha desarrollado el estudio, posteriormente se presenta la orientación temática de los estudios para ello se clasificaron tres grupos acorde a los diferentes ámbitos de la sustentabilidad, así mismo, se aborda desde los diversos enfoques metodológicos, a continuación, se muestra el análisis realizado, retomando las categorías mencionadas anteriormente.

\section{Espacio físico.}

Los artículos revisados se clasificaron de acuerdo con el espacio físico donde se desarrolla la actividad turística, para los cuales se dividieron en tres categorías que son destinos de playa, zonas urbanas y medios rurales. El espacio que destaca es los destinos de sol y arena, pues además de ser el más visitado y con más cantidad de hoteles, infraestructura y desarrollo; es el más estudiado, para esta categoría se cuentan con 19 investigaciones (Aguiar, 2017; Castillo et al., 2014; CEPAL, 2004; Ken, 2007; Lee \& Hsieh, 2016; Linares et al., 2019; Martínez \& Ruiz, 2006; Narváez \& Fernández, 2009; Ng et al., 2017; Oliveros \& Beltrán, 2018; Pérez et al., 2017; Pinar et al., 2014; Reed et al., 2006; Roberts \& Tribe, 2008; Salinas et al., 2008) enfocadas en evaluar la sustentabilidad con un énfasis en el cuidado de recursos naturales marinos, así mismo representa el mayor flujo de visitantes al año, por lo tanto, es importante enfatizar la creación de programas y políticas públicas que permitan tener un acercamiento a la sustentabilidad, pues al presentar un gran número de visitas al año y tener grandes y numerosos complejos turísticos es más evidente la degradación ambiental.
Posteriormente, se encuentra los estudios realizados en zonas urbanas con 15 investigaciones (Álvarez \& Valdés, 2016; Aranda, 2014; Arévalo et al., 2015; Blancas et al., 2017; Fraser et al., 2006; Gandara, 2000; González, 2004; Guillén et al., 2018; Ibañez, 2010; Mathew \& Sreejesh, 2017; Mendoza \& Hernández, 2017; Molinari, 2013; OMT, 2007; Pérez et al., 2009; Sánchez, 2007; N. C. Silva, 2009); en esta categoría principalmente se enfoca en impulsar la actividad turística, sin perder el patrimonio cultural en los edificios y fachadas, asimismo, representa un reto equilibrar el impacto que genera el turista y el impacto que se genera de forma natural derivado por la dinámica de las grandes urbes.

Para finalizar, las investigaciones enfocadas en zonas rurales son las menos estudiadas, con diez trabajos (Blancas et al., 2011; Blancas et al., 2017; Lonela, 2018; Márquez \& Toselli, 2015; Montero et al., 2017; OMT, 2000; Palafox et al., 2011; Víctor Pérez et al., 2009; Saragos, 2016; Silva, 2009); se enfoca en aprovechar los recursos culturales y ambientales, buscando el menor impacto posible y logrando el desarrollo en las comunidades donde se establece la actividad turisitica (Gráfico 3).

\section{Gráfico 1 Frecuencia por espacio físico}

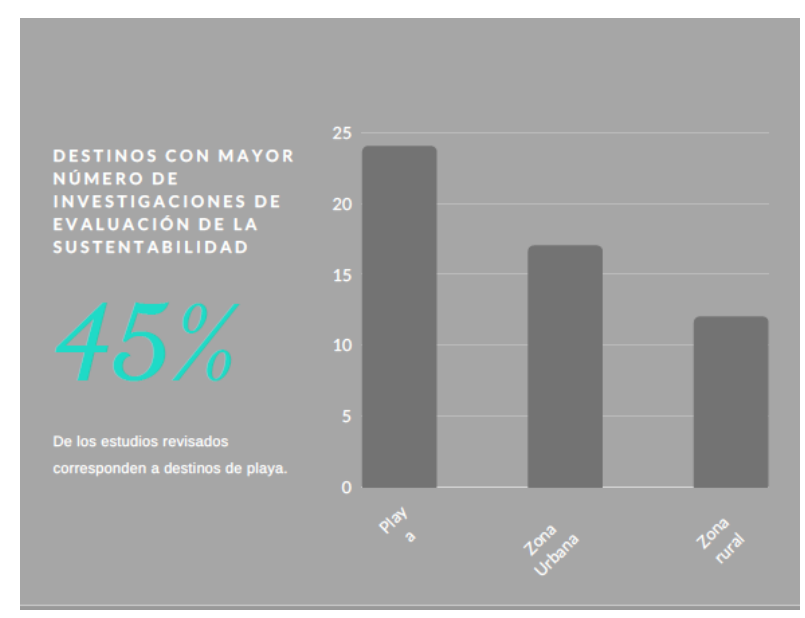

Fuente: Constructo del autor con base en datos recolectados. 


\section{Orientación temática}

En esta categoría se seleccionó la información de acuerdo con las tres esferas de la sustentabilidad (económico, ambiental y social). La categoría que destaca en este aparatado es aquella acorde la orientación económica, al establecer indicadores que destacan la importancia económica de la actividad, de esta forma promueven el modelo económico imperante a través de la validación verde la actividad y productos turísticos, así como la inclusión de los destinos turísticos de forma ventajosa en el mercado a través de certificaciones.

Esta información se encuentra representada en los siguientes autores (Aguiar, 2017; Blancas, LozanoOyola, González, Guerrero, \& Caballero, 2011; Blancas, Oyola, \& González, 2017; Ibáñez, 2018; Linares, Betancourt, \& Falcón, 2019; Mendoza \& Hernández, 2017; Molinari, 2013; Narváez \& Fernández, 2009; Serrado, Montoya - Restrepo, \& Cazares, 2018); la propuesta de la evaluación de la sustentabilidad se encuentra en la aplicación de indicadores relacionados a mejorar la competitividad de los destinos turísticos.

La siguiente categoría corresponde al enfoque orientado a la conservación de la naturaleza y la diversidad biológica, para ello destacan los trabajos de (Márquez \& Toselli, 2015; Pinar et al., 2014; Rossi, Charne, \& Comparato, 2018; Salinas et al., 2008; Sánchez, 2015; Saragos, 2016), donde se propone metodología enfocada principalmente en indicadores que permitan conocer la situación actual de degradación de sitios naturales para que posteriormente se puedan crear programas que impulsen la preservación y conservación de los recursos naturales.

Para finalizar, se han desarrollado diferentes estudios con énfasis social, reconociendo la importancia de los actores locales en el desarrollo de la actividad turística, (Fraser, Dougill, Mabee, Reed, \& Mcalpine, 2006; Gandara, 2000; Lee \& Hsieh, 2016; Lonela, 2018; Mendoza \& Hernández, 2017; Ng, Chia, Ho, \& Ramachandran, 2017; Pérez et al., 2017; Saragos, 2016), tomando en cuenta las distintas redes sociales y grupos de apoyo generado gracias a la actividad turística, y como de esta forma logran una comunidad organizada que permita tomar decisiones que impacten de manera positiva en el desarrollo de la comunidad sin poner en riesgo el crecimiento económico, la diversidad biológica y el bienestar social.

\section{Gráfico 4 Orientación temática de revisión de la} literatura

\section{Orientación temática}

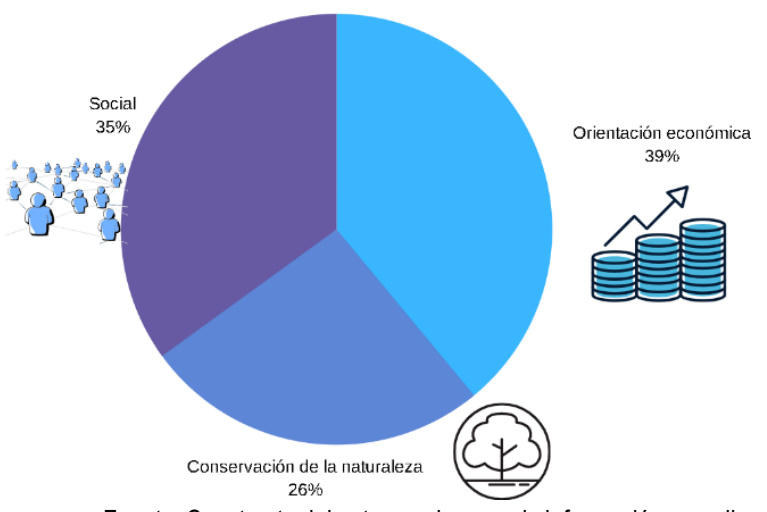

Fuente: Constructo del autor con base en la información recopila

Como se puede observar en el gráfico anterior (gráfico 4) existe un interés notable en aumentar el crecimiento económico a través de una validación verde de la actividad, pero es importante establecer metodologías que permitan detectar las áreas de oportunidad desde los diferentes ámbitos de la sustentabilidad y de esta manera tener un acercamiento a la sustentabilidad de los destinos turísticos, asimismo es importante incluir a los actores locales como punto clave de desarrollo.

\section{Enfoque metodológico}

La evaluación de la sustentabilidad se ha originado a través de distintos enfoques metodológicos, que de acuerdo con Speelman y colaboradores (2007) se clasifican en tres:

a) La evaluación de la sustentabilidad se centra en la generación de indicadores nacionales e internacionales, adecuado a contextos diversos, abordando temáticas ambientales y económicas, y en menor medida aquellos de índole social e institucional (llustración 2). Creando un índice que resume la información a un valor numérico, que hace poco viable 
una retroalimentación y establecer procedimientos para la planificación y toma de decisiones.

\section{Ilustración 2 Procedimiento para la}

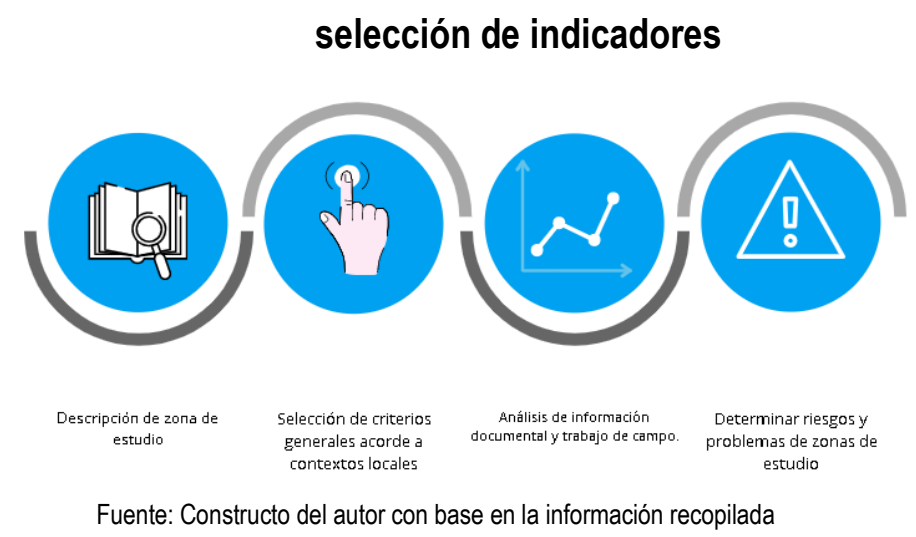

En este grupo encontramos el mayor número de estudios, retomando indicadores como los delineados por la Organización Mundial del Turismo (OMT) (2000), índice de desarrollo humano (IDH) (PNUD, 2018), Objetivos de desarrollo sustentable (ODS) (UNSD, 2016), los propuestos por la Comisión Económica de América Latina y el Caribe (CEPAL) (Schuschny \& Soto, 2009), entre otros; que permiten establecer las características de la zona de estudio y se aplican a criterios generales en contextos locales en específico, apoyado del análisis de información documental y trabajo de campo, a través de aplicación de entrevistas, encuestas, observación directa, entre otros.

Así mismo, los indicadores son seleccionados con base en la relevancia en la orientación temática, por ejemplo; en España Pérez et. al (2009); la clasificación se realizó acorde con la planificación y gestión del destino, así como la disponibilidad de datos. En ese sentido, Blancas, Oyola y González (2017) usaron indicadores de acuerdo a la tipología del turismo y la evolución del destino. Además, Blancas, LozanoOyola, González, Guerrero y Caballero (2011) retoma la caracterización de la zona de estudio, así como los puntos clave que establece la OMT en la definición de turismo sustentable. Existen autores como Álvarez y Valdés, (2016) que toma el Sistema de Información Turística de Asturias (SITA) como base de su investigación, dividiendo los indicadores en cuatro apartados: gestión sustentable, economía, socio/cultural y medio ambiente.

En Europa, la Organización Mundial del Turismo selecciona los indicadores para la región Kolašin, Montenegro (2007) trabajando con distintos grupos de expertos, buscando adecuar su metodología general a un caso en particular. En Asia, Mathew y Sreejesh (2017) toma en cuenta varias metodologías internacionales y de distintos autores para crear su propuesta de indicadores. Para finalizar, en Reino Unido Roberts y Tribe (2008) desde una perspectiva multisectorial toman los indicadores de la OMT, indicadores culturales (Craik) e indicadores sociales y económicos sustentables de Carribean Tourism Organisation.

En el continente americano, Suramérica destaca con una notable cantidad de estudios respecto al tema. Salinas, Navarro, Echarri y La O (2008) para la obtención del grado de sustentabilidad turística del destino en Cuba, realizaron la selección de indicadores ateniendo a las características de la información, su actualidad, confiabilidad y representatividad en el territorios; en este caso fueron seleccionados 24 indicadores basados en las Unidades de Gestión Turística (UGT) con tres apartados, ambiente, gerencia y comunidad; asimismo Pérez, et al. (2017), empezaron la selección de indicadores propuestas por la UGT, complementados con los propuestos por la OMT y la Asociación de Estados del Caribe (AEC).

En Argentina, Sánchez (2007) emplea un sistema de indicadores compuesto por cuatro dimensiones: ambiental, sociocultural, económica y políticoinstitucional, en contraste, en Villa Gesell, Argentina; la OMT (2000) determinó los riesgos, problemas y factores clave del sitio que en conjunto como investigación participativa, diversos expertos crearon indicadores. En Chile, Molinari (2013) retoma el Producto Interno Bruto (PIB), índices económicos nacionales, el índice de precios al consumidor (IPC), además de encuestas a pobladores, para la creación de una Matriz de Fortalezas, Oportunidades, Debilidades y Amenazas (FODA), conformando 24 indicadores biofísicos para la evaluación de la sustentabilidad. 
En el caso de Venezuela; Narváez y Fernández (2009) a partir de registros, entrevistas y observaciones se seleccionaron los indicadores a evaluar en el área de estudio. En Colombia, Oliveros y Beltrán (2018) se enfocan en evaluar la sustentabilidad de los prestadores de servicios turísticos, para ello, realizó tres fases, la primera es la observación y entrevistas, la aplicación del instrumento y para finalizar la tabulación y análisis de información; contando con 23 categorías para su análisis. En el Salvador, Márquez y Toselli (2015) toman la sustentabilidad desde la perspectiva del turismo y toma en cuenta la eficacia de gestión del sitio, beneficios sociales y económicos; así como el cuidado del patrimonio cultural.

Para finalizar, en México, Martínez y Ruiz (2006) realizaron investigación documental y campo; tomando indicadores nacionales propuestos por la Secretaría de Turismo (SECTUR); Sánchez (2007) realizó una evaluación de políticas públicas y a partir de ahí se definió el sistema de indicadores de turismo sustentable; Ibáñez (2010) toma en cuenta cinco organismos para definir sus propios indicadores, la Unión Mundial para la Naturaleza (IUCN), Instituto Nacional de Estadística y Geografía (INEGI), SECTUR, OMT y la AEC.

Además, se ha tomado como parte de la metodología la caracterización del destino turístico, considerando el origen del desarrollo turístico, servicios ambientales, tenencia de la tierra, uso de suelo, nivel de dependencia económica del turismo, empleo y calidad del trabajo, propiedades y control nacional 0 extranjero, tipo de turismo, transporte e infraestructura, legislación, institucionalidad, participación social y cultura del medio ambiente (CEPAL, 2004) y adaptaciones para aplicarlo a la hotelería, conformada por 30 indicadores (Aguiar, 2017). a) El segundo enfoque metodológico está conformado por aquellas propuestas que se construye a través de la elaboración de marcos de evaluación. Estos tienen diferentes etapas que parten de objetivos que sirven de guía para construir criterios e indicadores más específicos, por esa razón permite tener resultados más integrales.

En la revisión de la literatura se identificó el Modelo Presión, Estado, Respuesta (PER) impulsado por la Organización de la Cooperación y el Desarrollo Económico OCDE (OCDE,1998). En este sentido, el PER ha sido empleado en España, en especial la ciudad de Zaragoza (Aranda, 2014); en Argentina (Montero, Arrieta, \& Oyaga, 2017) crearon 12 indicadores compuestos por cinco dimensiones el ambiental, uso de territorio, socio-cultural, económica y político-administrativo. En México, existen estos estudios (Castillo, González, Campos, Velázquez, Ferman, García, Hernández, Laguna, \& Yeladaqui,, 2014; González, 2004; Guillén, Abreu, Toribio, \& Fernández, 2018; Palafox, Collantes, \& Zizumbo, 2011) toman como base la metodología antes mencionada y los Objetivos de Desarrollo Sustentable propuestos por la ONU.

Entorno a los marcos de evaluación se ha empleado el Marco para la Evaluación de Sistemas de Manejo de Recursos Naturales Incorporando Indicadores Sustentables (MESMIS) utilizado principalmente en sistemas agroecológicos ha sido adaptado para la evaluación en destino turísticos, entre ellos destacan los estudios realizados en México por (Arévalo et al., 2015; Ken, 2007).

b) Para finalizar este grupo está conformado por diversas metodologías que son aplicables a diferentes situaciones y sirven de guía para construir criterios e indicadores más específicos. 
En este grupo destacan los estudios que utilizaron el método bottom up que toma en cuenta las voces, opiniones y sentimientos de los habitantes del lugar así como de quien toma cotidianamente decisiones estratégicas en función del destino turístico, por lo tanto, se fundamenta en reunir diferentes sistemas que conforman un todo, tomando en cuenta elementos individuales que se van uniendo unos con otros hasta conformar un sistema terminado y complejo (Borioli, 2009; Vidal, 2017); en Reino Unido (Reed et al., 2006), en Canadá (Fraser et al., 2006) y México (Saragos, 2016).

Asimismo se encontraron diversos estudios donde se toma en cuenta a los actores sociales, tal es el caso de Malasia (Ng et al., 2017), China (Lee \& Hsieh, 2016) toma un proceso de jerarquía analítica compuesto por dos categorías: stakeholder y ambiente; en el primero toman en cuenta los actores sociales, tales como Organizaciones de la Sociedad Civil (OSC), gobierno, turismo, residentes y organizaciones comunitarias, por otro lado, en la segunda categoría se toman variables como pérdida de recursos naturales, tasa de degradación de ecosistemas, impacto ambiental de la comunidad, salud de la población humana y pérdida de recursos naturales, entre otros.

También se utilizó el método bola de nieve, en España, Lonela (2018), Canadá, Fraser et. al (2006); en Argentina, Gandara (2000) toma en cuenta a la comunidad local en todas las fases de desarrollo para la creación de indicadores. En Cuba Pérez et al. (2017) considera la elección de indicadores consultando a los stakeholders, además de aplicar a turistas y población local para poder identificar principios y valores a tomar en cuenta; para finalizar en México, Mendoza y Hernández (2017) y Saragos (2016) se toma como base la identificación de tres niveles que se encuentran conformado por la comunidad, territorio y gobierno; de esta forma se busca conocer las necesidades y a partir de ahí trabajar en conjunto.
En el esquema (llustración 3) anterior se puede visualizar con más detalle las metodologías utilizadas en los trabajos realizados, así como el lapso en los cuales ha incrementado el estudio de la evaluación de la sustentabilidad. Aquellos autores que retomaron la selección y adaptación de indicadores de acuerdo con el contexto, se incluye un recuadro con las categorías de análisis en las cuales se basaron para la construcción de su metodología.

\section{Validación de métodos}

Como parte de la propuesta de indicadores para evaluar la sustentabilidad es importante validar los indicadores; de acuerdo con la revisión previa destaca el Método Delphi, donde expertos en el tema, evalúan los indicadores de acuerdo con su relevancia, viabilidad, credibilidad, claridad y comparabilidad, tal es el caso, en China (Lee \& Hsieh, 2016) donde se consultaron 28 expertos en él tema, en Argentina (OMT, 2000) no llevan a cabo algún método en específico para la validación de indicadores, pero si toman en cuenta algunas características como pertinencia del indicador, disponibilidad de datos, comprensión, credibilidad, comparabilidad y valor predictivo, en Cuba (Pérez et al., 2017; Pinar et al., 2014) utilizan el Goal Programming Synthetic Indicator (GPSI) que permite escoger las variables que ofrece mejor solución a la problemática y en México destaca las aportaciones de Ibáñez (2018) y Mendoza y Hernández (2017).

De forma complementaria, algunos estudios se han apoyado de herramientas estadísticas para poder establecer ponderaciones en los indicadores, entre ellos destacan el indicador sintético de distancia (DP) (Blancas et al., 2011; Linares et al., 2019; Pérez et al., 2009), el análisis envolvente de datos DEA (Pérez et al., 2009; Pinar et al., 2014), Indicador dinámico diferencial (Blancas et al., 2017) y el índice sumatorio ponderado (Oliveros \& Beltrán, 2018). 


\section{Ilustración 3 Mapa de estudios de evaluación de la sustentabilidad}

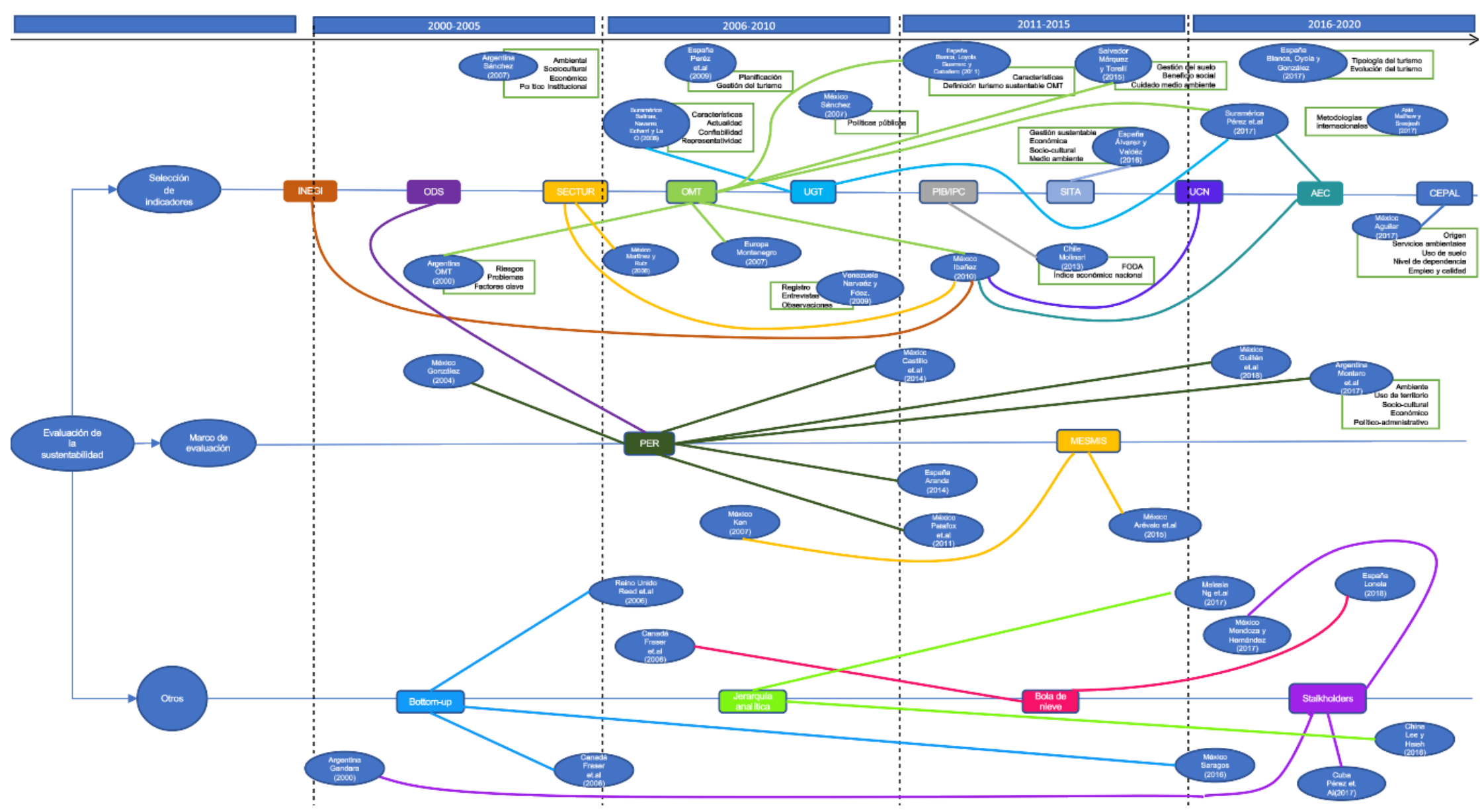

Fuente: Constructo del autor con base en la información recopilada en la revisión de la literatura 


\section{CONCLUSIONES:}

Derivado del análisis mencionado es posible reconocer la multiplicidad de estudios que se han realizado para la evaluación de la sustentabilidad del turismo, a través de diversos enfoques y corrientes teóricas; respecto a la orientación temática predominan los estudios enfocados en incrementar el número de visitantes y posicionar al destino como sustentable, en conjunto mejora la competitividad del destino turístico y aumenta ganancias económicas, que principalmente son acaparadas por los corporativos de la industria turística. En menor, frecuencia se encuentran trabajos que se especializan en la conservación de la naturaleza y en incluir a los actores sociales en la toma de decisiones y el proceso de desarrollo de la actividad turística.

No obstante, a pesar de que el concepto de sustentabilidad se encuentra basado en el ámbito ambiental, económico y social; pocos estudios construyeron indicadores y marcos de evaluación soportado en los tres pilares, en la mayoría de los estudios revisados se da prioridad en uno o dos ámbitos. Por ese motivo, es importante plantear metodologías que retomen el término de forma compleja y transdiciplinaria.

Del mismo modo, en el enfoque metodológico predominan los trabajos que refieren a la construcción de indicadores conformados por bases de datos y modos estadísticos, enfocados principalmente en rentabilidad económica y uso de recursos, excluyendo otros aspectos de carácter social sin tomar en cuenta herramientas cualitativas como las entrevistas y cuestionarios aplicados a la población para detectar las necesidades y condiciones en las cuales viven los habitantes. Además, la mayoría de los trabajos realizados toman como unidad de análisis destinos turísticos de litoral, esto es gracias a que los destinos de sol y playa son los de mayor demanda e importancia económica.
Así mismo, los marcos de evaluación referidos en la revisión de la literatura son aquellos propuestos por organismos internacionales como la CEPAL, que son adaptados a contextos locales y en algunos casos adaptados al turismo, tal es el caso del marco MESMIS. En el caso, de aquellos estudios que adaptan otras metodologías a la evaluación de la sustentabilidad, como el método Bottom Up, Jerarquía Analítica, y Bola de nieve, posicionan a los actores locales como pieza esencial en el planteamiento de políticas públicas.

Para finalizar, la validación de métodos más recurrente es el Método Delphi, con la validación de instrumentos por personas expertos en el tema. No obstante, también se apoyan de distintos modelos estadísticos que refuerce la veracidad y certeza de las metodologías.

Sin embargo, a pesar de estas valiosas contribuciones para la evaluación de la sustentabilidad en destinos turísticos, es evidente la ausencia de estudios orientados a comprender los alcances y limitaciones de la actividad con respecto al papel de los actores locales en los procesos de planificación y gestión de la actividad, la definición de indicadores cualitativos que conlleven a comprender la realidad, incluso abordar otros lugares de estudio más allá de los espacios tradicionales de sol y playa, tal es el caso de los Pueblos Mágicos, destinos poco estudiados en materia de sustentabilidad.

\section{REFERENCIAS:}

Aguiar, J. G. (2017). Indicadores de sostenibilidad para hoteles cinco estrellas medición dimensional social, cultural, ambiental, política y económica. SATHIRI, 12, 107-126.

Álvarez, R., \& Valdés, L. (2016). Selección e indicadores para la evaluación del desarrollo sostenible de un destino turístico. Aplicación al municipio de Gijón. ROTUR, Revista de Ocio y Turismo, 11(1888-6884), 12-21. 
Aranda, S. M. (2014). TURISMO SOSTENIBLE EN LA CIUDAD DE ZARAGOZA. Geographicalia, 99132.

Arévalo, G. J., Guerrero, H. R., \& Ortiz, C. F. (2015). Evaluación de la sustentabilidad del turismo de salud en michoacán, méxico. En $20^{\circ}$ Encuentro Nacional sobre Desarrollo Regional en México (pp. 1-23).

Beaucage, P. (2007). El desarrollo sustentable : crítica del discurso y de las prácticas. Estudios del hombre, (22), 41-70.

Blancas, F J, Lozano-Oyola, M., González, M., Guerrero, F. M., \& Caballero, R. (2011). How to use sustainability indicators for tourism planning : The case of rural tourism in Andalusia ( Spain ). Science of the Total Environment, 412-413, 2845.

https://doi.org/10.1016/j.scitotenv.2011.09.066

Blancas, Francisco Javier, Oyola, M. L., \& González, M. (2017). A dynamic sustainable tourism evaluation using multiple benchmarks $A$ DYNAMIC SUSTAINABLE TOURISM EVALUATION USING MULTIPLE. Journal of Cleaner Production. https://doi.org/10.1016/j.jclepro.2017.10.295

Borioli, G. (2009). Escribir para compartir. El modelo bottom-up y el aprendizaje con los pares. Praxis educativa, XIII, 101-105.

Castillo, L., González, M. A., Campos, B., Velázquez, D., Ferman, J. L., García, A., ... Yeladaqui, M. (2014). Modelo de indicadores para la evaluación y monitoreo del desarrollo sustentable en la zona. Perspectiva Geográfica, 19(2), 309-330.

CEPAL. (2004). Comercio de Servicios Ambientales Vinculados al Turismo y Revisión de la Sostenibilidad del Turismo en Tres Casos de Estudio.

César, A. A., Anaya, L. A., \& Ruiz, F. J. (2017). El turismo y la sustentabilidad real. Revista Anais Brasileiros de Estudos Turísticos ABET, 7, 8-17.

Fraser, E. D. G., Dougill, A. J., Mabee, W. E., Reed, M., \& Mcalpine, P. (2006). Bottom up and top down: Analysis of participatory processes for sustainability indicator identification as a pathway to community empowerment and sustainable environmental management. Journal of Environmental Management, 78, 114-127. https://doi.org/10.1016/j.jenvman.2005.04.009

Gandara, J. M. (2000). La Sostenibilidad de los Destinos Turísticos Urbanos, 1-29.

González Damián, A. (2004). Complejidad, desarrollo sostenible y turismo. Reflexiones sobre el modelo P-E-R y la necesidad de aproximaciones transdisciplinarias. En VI Congreso Nacional de Investigación Turística (p. 12).

Guillén, A., Abreu, J. L., Toribio, Y., \& Fernández, E. (2018). Indicadores de desarrollo sustentable y su aplicación. International Journal of Good Conscience., 13(1), 178-236.

Ibañez, R. M. (2010). Sustentabilidad e Indicadores de Desarrollo Turístico en México. Revista de investigación en turismo y desarrollo rural., 3.

Ibáñez, R. M. (2018). Medición de la sustentabilidad turística en una pequeña localidad costera a través de indicadores, barómetros y consulta a expertos. Nova Scientia, 10(2), 475-523. https://doi.org/doi.org/10.21640/ns.v10i21.1416

Ken, H. (2007). Análisis del turismo sustentable en Mahahual a través de un sistema de indicadores.

Lee, T. H., \& Hsieh, H. P. (2016). Indicators of sustainable tourism: A case study from a Taiwan's wetland. Ecological Indicators, 67, 779-787.

https://doi.org/10.1016/j.ecolind.2016.03.023

Linares, H. L., Betancourt, M. E., \& Falcón, M. C. (2019). Sostenibilidad y Desarrollo Local: Procedimientos para la Evaluación Integrada de los Destinos Turísticos. Rosa dos Ventos Turismo e Hospitalidade, 11. https://doi.org/DOI: http://dx.doi.org/10.18226/21789061.v11i1p84

Lonela, D. (2018). Estudio de la sostenibilidad en destinos turísticos a través de la participación comunitaria: el caso de la Costa del Sol. Boletín de la Asociación de Geógrafos Españoles.

Márquez, G., \& Toselli, C. (2015). Criterios de sustentabilidad en areas naturales rurales 
orientados al desarrollo local y la aplicación de buenas prácticas en turismo. Anuario de investigación USAL, 133-134.

Martínez, O. C., \& Ruiz, J. G. (2006). Las Particularidades de la Agenda 21 para el Turismo Mexicano. Un Análisis de la Aplicación del Sistema de Indicadores de Sustentabilidad en el Municipio de Playas de Rosarito, B.C. México. Gestión Turística, 9-29.

Mathew, P. V., \& Sreejesh, S. (2017). Impact of responsible tourism on destination sustainability and quality of life of community in tourism destinations. Journal of Hospitality and Tourism Management, 31, 83-89. https://doi.org/10.1016/j.jhtm.2016.10.001

Mendoza, J., \& Hernández, R. (2017). Una propuesta metodológica para la medición de la sostenibilidad del turismo a escala local *. Cuadernos Económicos. https://doi.org/https://doi.org/10.32796/cice.2017 .93 .6148

Molinari, G. (2013). Indicadores de sustentabilidad de la actividad turística en la ciudad de la plata. En XII Jornadas Nacionales de Investigación en turismo.

Montero, H., Arrieta, J., \& Oyaga, R. (2017). Valoración de la sostenibilidad turística del municipio de Usiacuri mediante la implementación de un sistema de indicadores. Seminario Internacional de Ciencias Ambientales SUE-Caribe, 1, 178180.

Narváez, M., \& Fernández, G. (2009). Indicadores de Competitividad para Destinos Turísticos en el Marco de la Sostenibilidad: Un Análisis Aplicado a la Península de Paraguaná. Revista Venezolana de Análisis de Coyuntura, (January).

Ng, S. I., Chia, K. W., Ho, J. A., \& Ramachandran, S. (2017). Seeking tourism sustainability - A case study of Tioman Island, Malaysia. Tourism Management, $\quad 58, \quad 101-107$. https://doi.org/10.1016/j.tourman.2016.10.007

Nieves, M. (1998). En torno al concepto de sustentabilidad. En Género, Medio ambiente y Sustentabilidad del Desarrollo (p. 400). Santiago de Chile: Naciones Unidas.
OCDE. (2000). Towards Sustainable Development: Indicators to measure progress. Rome Conference. OECD Rome Conference.

Oliveros Ocampo, C. A., \& Beltrán Vargas, J. E. (2018). Evaluación de la sustentabilidad de los prestadores de servicios turísticos del Parque Nacional Natural Tayrona en el departamento de Magdalena, Colombia. Cuadernos de Geografía: Revista Colombiana de Geografía, 27(1), 100 117. https://doi.org/10.15446/rcdg.v27n1.61589

Olivier, B., Rodríguez, A. L., López, R., Cañedo, R., \& Valera, M. Á. (2005). Marginación, contaminación y sustentabilidad en el desarrollo turístico de Punta Diamante en la Ciudad de Acapulco, México. Revista Latinoamericana el Ambiente y las Ciencias.

OMT. (2000). Taller de indicadores de sostenibilidad en turismo para los países de Sudamérica. Villa Gesell, Argentina.

OMT. (2007). Sustainable Tourism Indicators and Destination Management. OMT.

OMT. (2019). Definicion turismo sostenible. Recuperado de http://sdt.unwto.org/es/content/definicion

Palafox, A., Collantes, A., \& Zizumbo, L. (2011). Indicadores de Desarrollo Local para el Turismo Rural. Rosa dos Ventos -Turismo e Hospitalidade, 3, 133-140.

Pérez, Víctor E., Blancas, F. J., González, M., Guerrero, F. M., Lozano, M., Pérez, F., \& Caballero, R. (2009). Evaluación de la Sostenibilidad del Turimo Rural Mediante Indicadores Sintéticos. Revista Investigación Operacional, 30(1), 40-51.

Pérez, Victor Ernesto, Hernandez, A., Guerrero, F., León, M. A., da Silva, C. L., \& Caballero, R. (2017). Measuring the sustainability of Cuban tourism destinations considering stakeholders' perceptions. International Journal of Tourism Research, 19(3), 318-328. https://doi.org/10.1002/jtr.2114

Pinar, U. De, Técnicas, F. D. C., Matemáticas, D. De, Río, P., Olavide, U. P. De, Económicas, F. D. C., ... Caballero, R. (2014). La sostenibilidad de los 
destinos cubanos de turismo de naturaleza : un enfoque cuantitativo Víctor Pérez. Tourism \& Management Studies, 10(2).

PNUD. (2018). Índices E Indicadores De Desarrollo Humano. Programa de las Naciones Unidaspara el Desarrollo(PNUD).

Reed, M. S., Fraser, E. D. G., \& Dougill, A. J. (2006). An adaptive learning process for developing and applying sustainability indicators with local communities. Ecological Indicators, 9. https://doi.org/10.1016/j.ecolecon.2005.11.008

Reul, A. L. H., Febles-patrón, J. L., \& Nava-galindo, V. A. (2015). La contaminación fecal en cenotes de interés turístico y recreacional del estado de Yucatán Fecal contamination in sinkholes of touristic and recreational interest in Yucatan.

Roberts, S., \& Tribe, J. (2008). Sustainability Indicators for Small Tourism Enterprises - An Exploratory Perspective Sustainability Indicators for Small Tourism Enterprises - An Exploratory Perspective. Journal of Sustainable Tourism, (December 2014), 37-41. https://doi.org/10.1080/09669580802159644

Romero, S. (2010). Calidad del Agua para Actividades Recreativas del Río Hardy en la Región Fronteriza México-Estados Unidos Water Quality for Recreational Activities of the Hardy River in the Mexican-U . S . Border Region, 21(5), 69-78. https://doi.org/10.1612/inf.tecnol.4357it.09

Rossi, E., Charne, U., \& Comparato, G. (2018). Turismo y ambiente. Repensar la actividad turística desde la sustentabilidad. (Editorial).

Salinas, E., Navarro, E., Echarri, M., \& La O, J. A. (2008). Metodologias para la Evolución de la Sustentabilidad Territorial: El Uso de Indicadores en Destinos Turisticos de Cuba. Boletín de la Real Sociedad Geografica CXLIV, 2008(1991).

Sánchez, D. C. (2007). UN SISTEMA DE INDICADORES TURÍSTICOS BÁSICOS: PRIMERA APROXIMACIÓN.

Sánchez, D. C. (2015). Sistemas de Indicadores de Sustentabilidad Turística para el desarrollo regional: una cuestión geográfica. Revista geografica, 1083.
Saragos, J. (2016). Desarrollo de indicadores de sustentabilidad turística aplicado a una comunidad indígena de la Selva Lacandona.

Schuschny, A., \& Soto, H. (2009). Guía metodológica Diseño de indicadores compuestos de desarrollo sostenible Andrés Schuschny. Santiago de Chile.

Serrado, A.-M., Montoya - Restrepo, L.-A., \& Cazares, I. (2018). Análisis de la sostenibilidad y competitividad turística en Colombia. Gestión y Ambiente, 21(1), 99-109.

Silva, N. C. (2009). Sistema de indicadores de sustentabilidade do desenvolvimento do turismo: um estudo de caso do município de Areia - PB Sustainability indicators system of tourism development: a case study os the municipality of Areia - PB Sistema de indicadores de sost. Revista Brasileira de Pesqueisa em Turismo, 10(3), 475-496.

Speelman, E., Astier, M., \& Galván, Y. (2007). Sistematización y análisis de los estudios de caso MESMIS: lecciones para el futuro. Fundación Instituto de Agricultura Ecológica y Sustentable (Vol. 1).

UNAM. (2020). EI marco MESMIS. Recuperado el 15 de mayo de 2020, de http://www.mesmis.unam.mx/

UNSD. (2016). Marco de indicadores mundiales para los Objetivos de Desarrollo Sostenible y metas de la Agenda 2030 para el Desarrollo Sostenible.

Vidal, D. F. (2017). Aplicación de los modelos "bottomup" de toma de decisión en los sistemas de producción pesquera artesanal a pequeña escala: el caso de las Reservas Marinas de Interés Pesquero de Os Miñarzos y Ría de Cedeira (Galicia).

Zizumbo, L., \& Monterroso, N. (2017). Sustentabilidad, turismo y hegemonía: Génesis y desarrollo de la retórica sobre el turismo sustentable. En Repensando el turismo sustentable (pp. 55-104). 